\title{
DIRECT DETERMINATION OF THE INTENSITY OF PICOSECOND LIGHT PULSES BY TWO-PHOTON ABSORPTION
}

\author{
A. PENZKOFER and W. FALKENSTEIN \\ Physik-Department der Technischen Universität München, \\ Munich, Fed. Rep. Germany
}

Received 15 February 1976

\begin{abstract}
The correspondence between energy transmission and input peak intensity of light pulses in two-photon absorbing media is used for intensity determination. The two-photon absorption coefficients for $\mathrm{CH}_{2} \mathrm{~J}_{2}$ at $5300 \mathrm{~A}$ and for rutile in the range between $5300 \AA$ and $6260 \AA$ are determined. Calibration curves for the intensity determination by transmission measurements are presented.
\end{abstract}

The conventional determination of the peak intensity of picosecond light pulses is time consuming and difficult: the energy, the duration and the beam crosssection of the light pulses must be measured simultaneously. For accurate intensity determinations the temporal and spatial pulse shape has to be known. A straightforward method of measuring the peak intensity of picosecond light pulses by determining the nonlinear transmission of light through a saturable absorber has been described previously [1]. This method is limited to the absorption region of the specific bleachable dye.

In this letter a new technique for the determination of the peak intensity of picosecond light pulses is presented. The intensity dependence of the energy transmission of a light pulse through a two-photon absorbing sample is applied in this technique. Calibration curves for the intensity determination by energy transmission measurements are presented in the spectral range between $5300 \AA$ and $6260 \AA$. A rutile crystal [2] and the liquid methylene iodide $\left(\mathrm{CH}_{2} \mathrm{~J}_{2}\right)$ were used for the two-photon absorption measurements.

The new technique of intensity determination by monitoring the two-photon absorption has several advantages. $i$ ) Two-photon absorption is a very rapid nonlinear process. No transient effects occur for picosecond light pulses. ii) The measured energy transmission is independent of the pulse duration and the beam radius (see below). iii) No absolute signal values have to be measured. iv) The energy transmission is only slightly dependent on the temporal and spatial pulse shape. v) The measurement is easy and can - in most practical cases - be carried out with a small part of the picosecond light pulse. vi) The method can be applied in the infrared, visible and ultraviolet range by choosing proper two-photon absorbing media.

The two-photon absorption of light is described by the differential equation

$\frac{\partial I(r, t, z)}{\partial z}=-\alpha^{(2)} I^{2}(r, t, z)$,

where $I$ is the light intensity, $r$ is the radial coordinate for the light beam (radial symmetry is assumed), $t$ represents the time, $z$ describes the propagation distance in the sample, and $\alpha^{(2)}$ is the two-photon absorption coefficient. The solution of eq. (1) has the form:

$$
I(r, t, z)=\frac{I(r, t, 0)}{1+\alpha^{(2)} z I(r, t, 0)} .
$$

The input intensity $I(r, t, 0)$ is factorized into the peak intensity $I_{0}$ and a form factor $s\left(r / r_{0}, t / t_{0}\right)$ :

$I(r, t, 0)=I_{0} \times s\left(r / r_{0}, t / t_{0}\right)=I_{0} \times s\left(r^{\prime}, t^{\prime}\right)$.

$r_{0}$ and $2 t_{0}$ are characteristic values (half widths or $1 / \mathrm{e}$ values) of the beam radius and of the pulse duration, respectively.

The energy transmission $T_{E}$ is defined by the ratio of the transmitted light energy to the input light energy 


$$
T_{\mathrm{E}}=\frac{\int_{0}^{\infty} 2 \pi r \mathrm{~d} r \int_{-\infty}^{\infty} \mathrm{d} t I(r, t, l)}{\int_{0}^{\infty} 2 \pi r \mathrm{~d} r \int_{-\infty}^{\infty} \mathrm{d} t I(r, t, 0)}
$$

Together with eqs. (2) and (3) we find

$$
\begin{aligned}
T_{\mathrm{E}} & =\frac{(1-R)^{2}}{1-\int^{2}} \frac{\int_{0}^{\infty} r^{\prime} \mathrm{d} r^{\prime} \int_{-\infty}^{\infty} \mathrm{d} t^{\prime}}{1} \\
& \times \frac{s\left(r^{\prime}, t^{\prime}\right) /\left[1+\alpha^{(2)} l(1-R) I_{0} s\left(r^{\prime}, t^{\prime}\right)\right]}{\int_{0}^{\infty} r^{\prime} \mathrm{d} r^{\prime} \int_{-\infty}^{\infty} \mathrm{d} t^{\prime} s\left(r^{\prime}, t^{\prime}\right)} .
\end{aligned}
$$

The loss of light by reflections is taken into account by the fraction $(1-R)^{2} /\left(1-R^{2}\right)$ and the factor $(1-R)$. Eq. (4) shows that the energy transmission does not depend on the pulse duration $2 t_{0}$ and the beam radius $r_{0} . T_{\mathrm{E}}$ is a function of the input peak intensity $I_{0}$, the pulse profile $s\left(r^{\prime}, t^{\prime}\right)$, the sample length $l$, the reflection factor $R$, and the two-photon absorption coefficient $\alpha^{(2)}$.

Eq. (4) represents a one-to-one correspondence between $T_{\mathrm{E}}$ and $I_{0}$ for fixed parameters of $s\left(r^{\prime}, t^{\prime}\right), l, R$ and $\alpha^{(2)}$. We assume the shape of our picosecond light pulse to be approximately gaussian [3] . Later we will show that slightly different pulse shapes do not affect the intensity determination significantly. The parameters $l, R$ and $\alpha^{(2)}$ are constants for a specific experimental arrangement. In this paper we carefully determined $\alpha^{(2)}$ for two materials. The knowledge of $\alpha^{(2)}$ enabled us to present calibration curves for definite values of sample length $l$, reflection loss $R$ and pulse shape $s\left(r^{\prime}, t^{\prime}\right)$.

The experimental setup is depicted in fig. 1. A modelocked $\mathrm{Nd}$-glass laser generates pulse trains of picosecond light pulses [4]. Single picosecond light pulses were selected from the pulse trains with an electrooptical shutter. The pulse energy was increased to approximately $3 \mathrm{~mJ}$ in an $\mathrm{Nd}$-glass amplifier. The single light pulses had a duration of $\Delta t \approx 6 \mathrm{ps}(\mathrm{fwhm})$ and a spectral width of $\Delta \widetilde{\nu} \approx 3 \mathrm{~cm}^{-1}$ (fwhm). Approximately $20 \%$ of the energy of the laser light pulse at $\lambda=1.06$ $\mu \mathrm{m}$ was converted to the second harmonic at $\lambda=5300$ $\AA$. The fundamental light beam was then filtered out.

In a first set of experiments the energy transmission of the picosecond light pulses at $\lambda=5300 \AA$ was investigated. A rutile crystal of $1 \mathrm{~cm}$ length and a $1-\mathrm{cm}$ long cell containing the liquid $\mathrm{CH}_{2} \mathrm{~J}_{2}$ were used as two-photon absorbing samples. The $c$-axis of the crystal was oriented perpendicular to the light polarization vector and to the propagation direction. The input in-

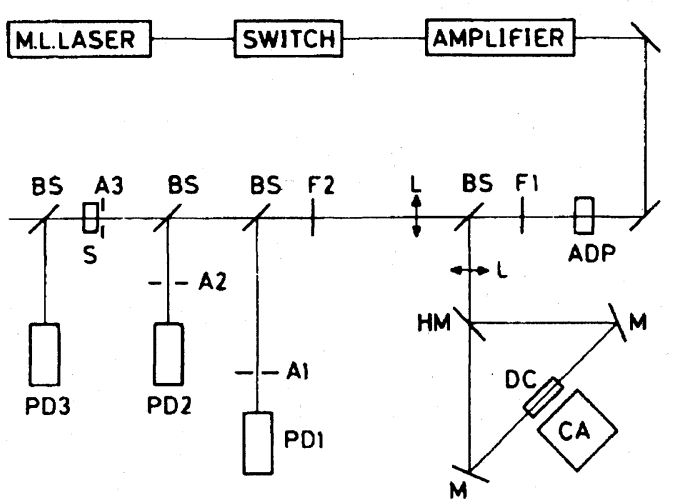

Fig. 1. Experimental setup for the determination of calibration curves for the intensity measurement. ADP, crystal for second harmonic generation; F1, filter to block off the fundamental laser light; L, lenses; BS, beam splitters; HM, 50\% mirror; $\mathrm{M}$, $100 \%$ mirrors; DC, two-photon absorbing dye cell for pulseduration measurement; CA, camera; F2, filters. A1 and A2 apertures for detection of beam radius with photo detectors PD1 and PD2. A3 aperture in front of sample S. PD2 measures input energy. The energy transmission is measured with the photodetectors PD2 and PD3.

tensity of the light pulses was varied with a lens and filters.

The peak intensity of the input pulses had to be determined by simultaneous measurements of the energy, the duration and the cross-section of the pulses. A small aperture A3 in front of the sample allowed only the central part of the light beam to enter the sample. The beam profile at the sample has a truncated gaussian shape. The beam radius $r_{0}$ (1/e value) of the incoming gaussian pulse was determined for every shot. It was calculated from the ratio of the energies that passed through the apertures $A 1$ and $A 2$. The width of the aperture $A 2$ is equal to that of $A 3$, the width of $A 1$ is smaller than that of A2. The energy transmission through the sample is measured with the photo detectors PD2 and PD3. The input energy of the light pulse was measured with the photo detector PD2. This detector was calibrated to absolute energy values with a pyroelectric energy meter. Care was taken that no background laser light or flash-lamp light could enter the energy meter. The flash-lamp light was filtered out and possible background laser light was eliminated with a saturable absorber. Simultaneously with the measurement of the input energy, the energy transmission and the beam radius, the pulse duration was measured. The two-photon fluorescence technique [5] in a colliding 
beam arrangement was used. The dye 9,10-diphenyl anthracene dissolved in cyclohexane was employed as two-photon absorber. The fluorescence pattern was photographed and the pulse durations deduced from the microdensitometer traces. From the measured data the two-photon absorption cross-sections for rutile and $\mathrm{CH}_{2} \mathrm{~J}_{2}$ at $\lambda=5300 \AA$ could be determined.

In a second experiment the two-photon absorption coefficient of rutile at $\lambda=6260 \AA$ was measured. The picosecond light pulses at this frequency were produced by stimulated Raman scattering of the second-harmonic light pulses in a cell of ethyl alcohol (length $5 \mathrm{~cm}$ ). The $\alpha^{(2)}$-value at $6260 \AA$ was found to be approximately half as large as the value at $5300 \AA$. The two-photon absorption behavior in the frequency range between $5300 \AA$ and $6260 \AA$ was checked with the picosecond two-photon spectroscopy technique described previously [6]. The second-harmonic light first generated the picosecond light continuum in water $(2 \mathrm{~cm}$ length) and then caused absorption of the continuum in the rutile crystal. The experimentally observed smooth absorption behavior indicates that $\alpha^{(2)}$ decreases monotonic in the range between $5300 \AA$ and $6260 \AA$.

In fig. 2 the measured energy transmission of (truncated gaussian) light pulses at $\lambda=5300 \AA$ is shown for rutile and $\mathrm{CH}_{2} \mathrm{~J}_{2}$ (sample length $1 \mathrm{~cm}$ ). The average pulse duration of the second harmonic light pulses was found to be $4.2 \mathrm{ps}$ (fwhm). The aperture $\mathrm{A} 3$ in front of the sample had a width of $0.72 \mathrm{~mm}$. The average 1 /e-radius of the gaussian light beams was found to be $0.29 \mathrm{~mm}$ in the experiments with rutile and 0.40 $\mathrm{mm}$ in the case of $\mathrm{CH}_{2} \mathrm{~J}_{2}$. The rutile crystal was antireflection coated and had a linear transmission of $T$ $=0.93(R=0.0335)$. The linear transmission through the $\mathrm{CH}_{2} \mathrm{~J}_{2}$ cell was $T=0.91(R \approx 0.045)$. The data points shown in the fig. 2 are averages over two to seven individual points. $\alpha^{(2)}$ was calculated with the help of eq. (4). The obtained $\alpha^{(2)}$ values are 1.4 $\times 10^{-8} \mathrm{~cm} / \mathrm{W}$ and $2.1 \times 10^{-9} \mathrm{~cm} / \mathrm{W}$ for rutile and $\mathrm{CH}_{2} \mathrm{~J}_{2}$, respectively. The curves in fig. 2 represent the energy transmissions for these $\alpha^{(2)}$-values. We note the good fit to the experimental points over the whole intensity range.

Absorption of laser light from excited states [7] was not detectêd. Calculations indicate that excited state absorption cross-sections up to $2 \times 10^{-17} \mathrm{~cm}^{2}$ have negligible effect on laser light absorption under our experimental conditions $\left(I_{0}<10^{10} \mathrm{~W} / \mathrm{cm}^{2}\right)$. From

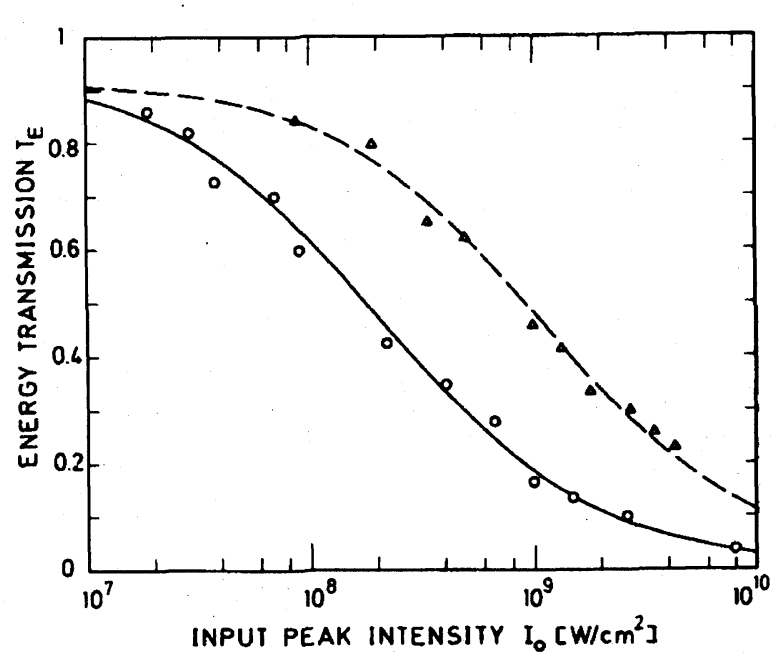

Fig. 2. Experimental energy transmission of light pulses at $5300 \AA$ versus input peak intensity. Width of the aperture A3 is $0.72 \mathrm{~mm}$. Circles and solid curve: rutile crystal with $c$-axis perpendicular to light pulse polarization and propagation direction, length $1 \mathrm{~cm}$; average $r_{0}=0.29 \mathrm{~mm}, R=0.0335, \alpha^{(2)}$ $=1.4 \times 10^{-8} \mathrm{~cm} / \mathrm{W}$. Triangles and dashed curve: $1 \mathrm{~cm}$ cell of $\mathrm{CH}_{2} \mathrm{~J}_{2}$, average $r_{0}=0.40 \mathrm{~mm}, R=0.045, \alpha^{(2)}=2.1 \times 10^{-9}$ $\mathrm{cm} / \mathrm{W}$.

the band structure of rutile no excited state absorption is expected [8]. The absorption spectrum of $\mathrm{CH}_{2} \mathrm{~J}_{2}$ is only known for $\lambda>2200 \AA[9]$.

The two-photon absorption cross-section of rutile at $\lambda=6260 \AA$ was determined in a way described above The pulse duration of the stimulated Raman light was not measured. It was assumed that in the saturation range it has the same duration as the exciting light pulse. The obtained $\alpha^{(2)}$.value is $6.5 \times 10^{-9} \mathrm{~cm} / \mathrm{W}$. In a simple theoretical model [10] the frequency dependence of the two-photon absorption coefficient of crystals is given by

$\alpha^{(2)}(\nu)=\gamma \sqrt{2 \nu-\nu_{g}}$,

where $\nu$ is the frequency of the laser light (in $\mathrm{cm}^{-1}$ ), $\nu_{\mathrm{g}}$ is given by the energy gap $E_{\mathrm{g}}$ of the crystal, $\nu_{\mathrm{g}}=$ $E_{\mathrm{g}} / h c$. In our case it is $E_{\mathrm{g}}=3.75 \mathrm{eV} \mathrm{[2]} \mathrm{and} \nu_{\mathrm{g}}=30244$ $\mathrm{cm}^{-1} \cdot \gamma=1.61 \times 10^{-10} \mathrm{~cm}^{3 / 2} / \mathrm{W}$ is determined from the measured $\alpha^{(2)}$ value at $5300 \AA$.

With the help of eq. (5) a two-photon absorption coefficient of $\alpha^{(2)}(6260 \AA)=6.7 \times 10^{-9} \mathrm{~cm} / \mathrm{W}$ is estimated. This value is in excellent agreement with the measured absorption coefficient. The absorption measurements with the picosecond continua verified a mo- 


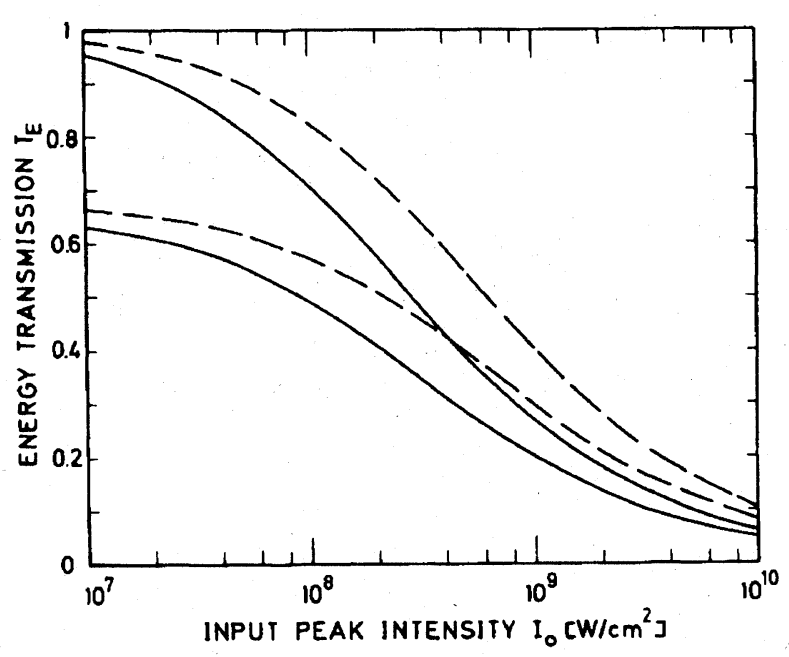

Fig. 3. Calculated energy transmission of gaussian light pulses versus input peak intensity. The sample is rutile of $1 \mathrm{~cm}$ length. Solid curves correspond to $\lambda=5300 \AA$, the dashed curves to $\lambda=6260 \AA$. The upper curves are calculated for a reflection factor of $R=0$ while in the lower curves $R$ is 0.207 and 0.196 for $\lambda=5300 \AA$ and $6260 \AA$, respectively.

notonic decrease of $\alpha^{(2)}$ at wavelengths longer than $5300 \AA$. These facts allow us to use eq. (5) for the calculation of $\alpha^{(2)}$ at wavelengths longer than $5300 \AA$, and with the aid of eq. (4), energy transmission curves versus laser peak intensity can be calculated. These curves are useful as calibration curves for the determination of laser intensity by energy transmission measurements over a substantial wavelength range.

In fig. 3 the calculated energy transmission of gaussian input light pulses versus the input peak intensity is depicted for the case of rutile at $\lambda=5300 \AA\left(\alpha^{(2)}\right.$ $=1.4 \times 10^{-8} \mathrm{~cm} / \mathrm{W}$, solid lines $)$ and $\lambda=6260 \AA\left(\alpha^{(2)}\right.$ $=6.5 \times 10^{-9} \mathrm{~cm} / \mathrm{W}$, dashed curves). A crystal length of $1 \mathrm{~cm}$ was used. The two upper curves belong to crystals with perfect anti-reflection coatings $(R=0)$. The two lower curves are valid for uncoated rutile crystals $(R=0.207$ at $\lambda=5300 \AA$ and $R=0.196$ at $\lambda=6260$ $\AA$ [11]). Fig. 3 indicates that rutile $1 \mathrm{~cm}$ in length is useful to measure directly the peak intensity of laser pulses in the range between $2 \times 10^{7}$ and $10^{10} \mathrm{~W} / \mathrm{cm}^{2}$. Outside this intensity region beam narrowers or filters have to be employed.

In fig. 4 the energy transmission of gaussian light pulses at $\lambda=5300 \AA$ through a cell of $\mathrm{CH}_{2} \mathrm{~J}_{2}$ is depicted versus input peak intensity. The curves apply to a cell length of $1 \mathrm{~cm}$ and a linear transmission of $T$

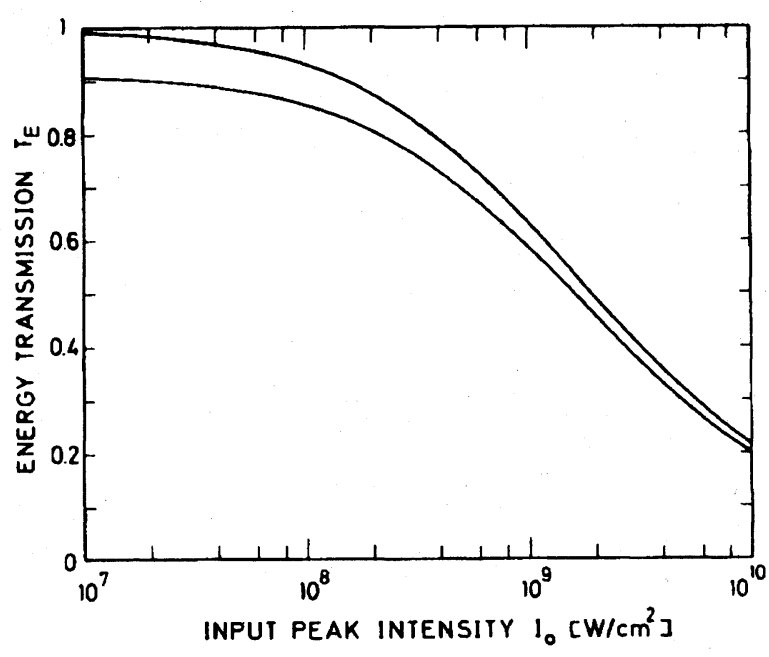

Fig. 4. Calculated energy transmission versus input peak intensity for gaussian light pulses. $\mathrm{CH}_{2} \mathrm{~J}_{2}$ at $\lambda=5300 \AA, \alpha^{(2)}$ $=2.1 \times 10^{-9} \mathrm{~cm} / \mathrm{W}$; sample length $l=1 \mathrm{~cm} . R=0$ (upper curve) and $R=0.045$ (lower curve).

$=91 \%(R=0.045$, lower curve $)$ and $T=1(R=0$, upper curve). The experimentally determined two-photon absorption coefficient of $\alpha^{(2)}=2.1 \times 10^{-9} \mathrm{~cm} / \mathrm{W}$ was used in the calculations.

The curves of figs. 3 and 4 are calibration curves for the determination of peak intensities by energy transmission measurements.

The effects of different pulse shapes on the energy transmission are shówn in figs. 5a and 5b. The energy transmissions for several transverse intensity distributions are depicted in fig. 5a. The considered shapes are $\mathrm{TEM}_{00}$-mode [gaussian profile, $s\left(r^{\prime}\right)=\exp \left(-r^{\prime 2}\right)$ ], $\mathrm{TEM}_{01}$-mode $\left[s\left(r^{\prime}\right)=r^{\prime 2} \exp \left(1-r^{\prime 2}\right)\right]$, diffraction pattern of a circular aperture $\left[s\left(r^{\prime}\right)=\left\{2 J_{1}\left(r^{\prime}\right) / r\right\}^{2}\right]$, and rectangular profile $\left[s\left(r^{\prime}\right)=1\right]$. The temporal shape is assumed to be gaussian. In fig. $5 b$ the effect of different temporal pulse shapes is presented for pulses with gaussian transverse distribution. Gaussian $\left[s\left(t^{\prime}\right)=\right.$ $\left.\exp \left(-t^{\prime 2}\right)\right]$, lorentzian $\left[s\left(t^{\prime}\right)=1 /\left(1+t^{\prime 2}\right)\right]$, hyperbolic secant $\left[s\left(t^{\prime}\right)=1 / \cosh \left(t^{\prime}\right)\right]$ and rectangular $\left[s\left(t^{\prime}\right)\right.$ $=1 \mathrm{]}$ pulse shapes are considered. The curves of fig. 5 indicate that pulse shapes similar to a gaussian pulse give slight deviations in the energy transmission curves. In other words the two-photon absorption technique is not very sensitive to the detailed pulse shape. We believe that the experimental method described here should be useful for intensity determinations over a wide frequency range in the visible part of the spectrum. 


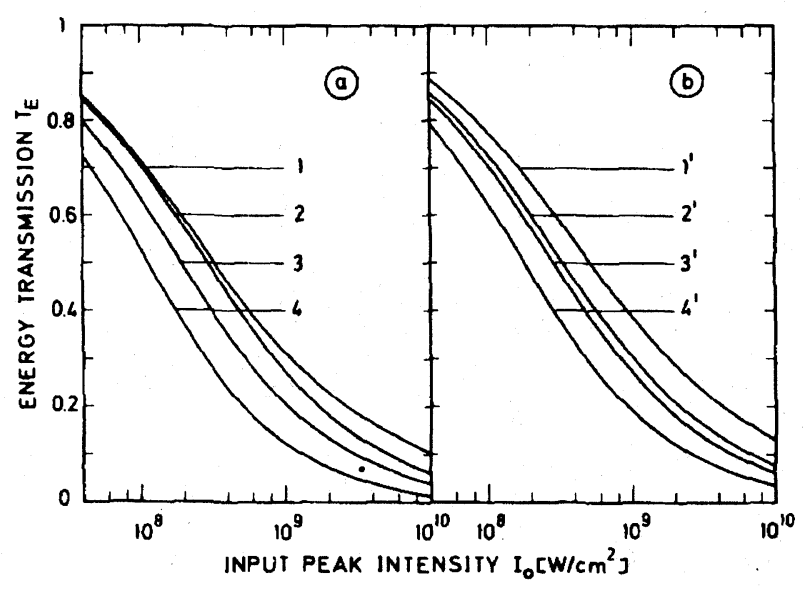

Fig. 5. Energy transmission versus input peak intensity for different pulse shapes.

a) The temporal pulse shape is gaussian while the transverse distribution has the following forms: diffraction pattern of a circular aperture (1), gaussian (2), a TEM 01 -mode (3) and rectangular (4).

b) The transverse pulse shape is gaussian. The temporal shapes are lorentzian ( $\left.1^{\prime}\right)$, hyperbolic secant $\left(2^{\prime}\right)$, gaussian ( $\left.3^{\prime}\right)$ and rectangular $\left(4^{\prime}\right)$. The parameters used are $\alpha^{(2)}=1.4 \times 10^{-8}$ $\mathrm{cm} / \mathrm{W}$, sample length $l=1 \mathrm{~cm}$ and reflection factor $R=0$.
The authors are very indebted to Professor Dr. W. Kaiser for many valuable discussions.

\section{References}

[1] A. Penzkofer, D. von der Linde and A. Laubereau, Opt. Comm. 4 (1972) 377.

[2] H.S. Waff and K. Park, Phys. Lett. 32A (1970) 109.

[3] D. von der Linde and A. Laubereau, Opt. Comm. 3 (1971) 279.

[4] A. Laubereau and W. Kaiser, Opto-Electronics 6 (1974) 1.

[5] J.A. Giordmaine, P.M. Rentzepis, S.L. Shapiro and K.W. Wecht, Appl. Phys. Lett. 11 (1967) 216.

[6] A. Penzkofer, W. Falkenstein and W. Kaiser, Appl. Phys. Lett. 28 (1976) 319.

[7] A. Penzkofer and W. Falkenstein, Opt. Comm. 16 (1976) 247.

[8] M. Cardona and G. Harbeke, Phys. Rev. 137A (1965) 1468.

[9] T.M. Lowry and R.A. Sass, J. Chem. Soc. 1 (1926) 622.

[10] R. Braunstein and N. Ockman, Phys. Rev. 134 (1964) A499.

[11] D.C. Cronemeyer, Phys. Rev. 87 (1952) 876. 